\title{
BMJ Open Protocol for a multicentre randomised feasibility trial evaluating early Surgery Alone In LOw Rectal cancer (SAILOR)
}

Dean A Harris, ${ }^{1}$ Kymberley Thorne, ${ }^{2}$ Hayley Hutchings, ${ }^{2}$ Saiful Islam, ${ }^{2}$ Gail Holland, ${ }^{2}$ Olivia Hatcher, ${ }^{3}$ Sarah Gwynne, ${ }^{3}$ lan Jenkins, ${ }^{4}$ Peter Coyne, ${ }^{5}$ Michael Duff, ${ }^{6}$ Melanie Feldman, ${ }^{7}$ Des C Winter, ${ }^{8}$ Simon Gollins, ${ }^{9}$ Phil Quirke, ${ }^{10}$ Nick West, ${ }^{10}$ Gina Brown, ${ }^{11}$ Deborah Fitzsimmons, ${ }^{12}$ Alan Brown, ${ }^{13}$ John Beynon ${ }^{1}$

To cite: Harris DA, Thorne $\mathrm{K}$, Hutchings $\mathrm{H}$, et al. Protocol for a multicentre randomised feasibility trial evaluating early Surgery Alone In LOW Rectal cancer (SAILOR). BMJ Open 2016;6:e12496. doi:10.1136/bmjopen-2016012496

- Prepublication history for this paper is available online. To view these files please visit the journal online (http://dx.doi.org/10.1136/ bmjopen-2016-012496).

Received 2 May 2016 Revised 5 October 2016 Accepted 7 October 2016

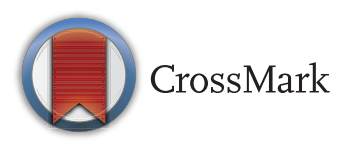

For numbered affiliations see end of article.

Correspondence to Professor Dean A Harris; WPMDAH2@cardiff.ac.uk

\section{ABSTRACT}

Introduction: There are 11500 rectal cancers diagnosed annually in the UK. Although surgery remains the primary treatment, there is evidence that preoperative radiotherapy (RT) improves local recurrence rates. High-quality surgery in rectal cancer is equally important in minimising local recurrence. Advances in MRI-guided prediction of resection margin status and improvements in abdominoperineal excision of the rectum (APER) technique supports a reassessment of the contribution of preoperative RT. A more selective approach to RT may be appropriate given the associated toxicity.

Methods and analysis: This trial will explore the feasibility of a definitive trial evaluating the omission of $\mathrm{RT}$ in resectable low rectal cancer requiring APER. It will test the feasibility of randomising patients to (1) standard care (neoadjuvant long course RT \pm chemotherapy and APER, or (2) APER surgery alone for cT2/T3ab N0/1 low rectal cancer with clear predicted resection margins on MRI. RT schedule will be 45 Gy over 5 weeks as current standard, with restaging and surgery after 8-12 weeks. Recruitment will be for 24 months with a minimum 12-month follow-up.

Objectives: Objectives include testing the ability to recruit, consent and retain patients, to quantify the number of patients eligible for a definitive trial and to test feasibility of outcomes measures. These include locoregional recurrence rates, distance to circumferential resection margin, toxicity and surgical complications including perineal wound healing, quality of life and economic analysis. The quality of MRI staging, RT delivery and surgical specimen quality will be closely monitored.

Ethics and dissemination: The trial is approved by the Regional Ethics Committee and Health Research Authority (HRA) or equivalent. Written informed consent will be obtained. Serious adverse events will be reported to Swansea Trials Unit (STU), the ethics committee and trial sites. Trial results will be submitted for peer review publication and to trial participants.

Trial registration number: ISRCTN02406823.

\section{Strengths and limitations of this study}

- A unique interventional study specific to low rectal cancer.

- Will explore the contribution of the modern abdominoperineal excision operation to cancer outcomes.

- Strict quality assurance (QA) processes for imaging, radiotherapy, surgery and pathology.

- Will establish if a future trial minimising radiotherapy use in low rectal cancer is feasible.

- Study is limited by short follow-up period.

\section{INTRODUCTION}

There are 11500 rectal cancers diagnosed annually in the UK. ${ }^{1}$ Surgery following preoperative long course radiotherapy (RT) has been the treatment of choice up until now, ${ }^{2}$ but recent advances in surgical technique and better MRI-guided prediction of resection margins now suggest that in patients with locally advanced low rectal cancer, surgery alone may be sufficient to minimise local recurrence. Given the associated longterm toxicity of RT, it is now time to reassess the appropriateness of preoperative RT as a standard treatment for this stage of cancer.

RT has been shown to increase postoperative complications, long-term toxicities and impaired quality of life (QoL), compared with surgery alone, and has not been shown to improve overall survival. There are marked differences between international recommendations for preoperative $\mathrm{RT}$ in rectal cancer (National Comprehensive Cancer Network (NCCN), ESMO, National Institute for Health and Care Excellence (NICE) $).{ }^{3-5}$ This is reflected by a survey of 28 countries $^{6}$ which reported 54 different treatment policies for rectal cancer, supporting the view that there is no current 
consensus on preoperative treatment. RT can be associated with significant harm, including acute and delayed severe toxicity, chronic pain, sexual dysfunction, small bowel obstruction, radiation enteritis, urinary and faecal incontinence and pelvic fractures. ${ }^{78}$ Subsequent surgery is associated with increased morbidity and wound complications after $\mathrm{RT}^{9}$ and reduced QoL. ${ }^{10} \mathrm{RT}$ can induce complete tumour regression but there remains disparity between complete clinical response rates and true complete pathological response rates such that non-operative management is not widely practised. ${ }^{11}$

The role of high-quality surgery in rectal cancer is well known. An involved surgical resection margin is the strongest predictor of locoregional recurrence and reduced survival. ${ }^{12}$ Low rectal cancer often requires an abdominoperineal excision (APE) procedure, which sacrifices the rectum and anus necessitating a permanent colostomy. National Bowel Cancer Audit suggests that this procedure remains in common use $(23.8 \%$ of all rectal cancer operations in 2006/2007 and 2011/ 2012 audit reports). ${ }^{13} 14$

Recent developments in refining the present technique for conduct of APE are based on recognition of the need for wide excision of the anal sphincter and pelvic floor in appropriate patients as defined by MRI. ${ }^{15} 16$ The Low Rectal Cancer National Development Programme (LOREC) ran between 2011 and 2014 and was developed to train colorectal surgeons in appropriate APE technique to improve outcomes. ${ }^{17}$ This so-called extralevator approach has been shown to significantly reduce rates of margin involvement and intraoperative tumour perforation over standard APE surgery ${ }^{18}$ yet has not been scrutinised in the absence of preoperative RT.

It is notable that the studies used to inform current guidelines which advocate preoperative RT had little quality control over surgical technique. For example, the German Rectal Cancer Study Group ${ }^{19}$ described no quality control; the EORTC 229021 trial $^{20}$ reported that just $36 \%$ patients had surgery in the TME plane. Although the recent MRC-CR07 study encouraged use of TME techniques, only $52 \%$ of patients had surgery in this optimal plane, which decreased to just $24 \%$ for APE. ${ }^{21}$ Future studies should strictly monitor surgical quality control as a defined end point.

Equally, high-quality preoperative MRI (notably absent in the chemoradiotherapy trials of Sauer $e t a l^{19}$ and Bosset $e t a l^{20}$ that define current guidelines) has been crucial to improved APE surgery through defining the relationship between the tumour and the predicted resection margin. Preoperative MRI assessment of the circumferential resection margin status is the most important factor predicting risk of local recurrence and survival. $^{22} 23$

There is growing evidence to suggest that a highly selective approach to the use of neoadjuvant RT may be more appropriate than widespread application and the need for further research is apparent. While many would consider T4 disease and those with significant nodal burden to be indications for neoadjuvant therapy the consensus is less apparent in the case of early T3 node negative tumours. RT is $80 \%$ more likely to be used for abdominoperineal excision of the rectum (APER) versus anterior resection (OR $0.20,95 \%$ CI 0.18 to $0.23, \mathrm{p}<0.01$; $\mathrm{P}$ Quirke, personal communication). This is more likely to be as long course RT, as national audit shows that short course RT $(5 \times 5)$ is rarely used in Europe for this indication. A more selective policy towards neoadjuvant treatment depends on highly accurate prediction of those patients in which there is a potentially low risk of local recurrence. This challenge in selecting subgroups with stage II and III disease who would least benefit from neoadjuvant treatment is evidenced by the wide variation in the use of preoperative RT in the Welsh Bowel Cancer Audit. ${ }^{24}$ This showed that the two units in Wales, UK achieving the lowest circumferential margin involvement rates (both 3\%) administered neoadjuvant therapy to a diverging $30 \%$ and $86 \%$ of patients, respectively. Trials are urgently required to address this variation in practice.

\section{METHODS AND ANALYSIS}

This is a prospective 1:1 randomised surgical intervention multicentre feasibility trial (2b exploration, IDEAL recommendation) studying the omission of preoperative long course RT in resectable locally advanced low rectal cancer requiring APER for oncological reasons. Recruitment is for 2 years with 12-month postsurgery follow-up. The feasibility trial will determine the willingness of participants and their clinicians to recruit to the study together with testing the ability to collect study data and its quality. It was considered important to determine these factors before launching the definitive trial as it challenges current recommendations that patients are considered for preoperative RT in locally advanced rectal cancers involving the lower third of the rectum. The feasibility trial will mirror the definitive trial design to test all aspects of trial conduct. All patients will be assessed by an MRI scan as per protocol.

\section{Trial groups}

Participants in this feasibility trial will be randomly assigned using a computerised randomisation system available as an internet-based application administered by Swansea Trials Unit (STU), Swansea University. Consenting patients will be randomised with equal probability to the intervention and control arms.

\section{Intervention arm: 'early surgery'}

Participants randomised to receive early high-quality APER. No preoperative RT treatment will be given to these patients. Standard adjuvant chemotherapy after surgery may be administered if indicated. Adjuvant postoperative pelvic chemoradiation will be given if 
histopathological examination shows that the circumferential resection margin is $\leq 1 \mathrm{~mm}$.

\section{Comparative arm: 'standard therapy'}

Standard preoperative long course RT will be given to participants. RT will be given as $45 \mathrm{~Gy}$ in 25 daily fractions over 5 weeks. Intensity modulated radiotherapy (IMRT) is permitted. Concurrent chemotherapy can be given, if required, in accordance with standard care. Participants will proceed to APER 8-12 weeks following RT completion.

\section{Adjuvant chemotherapy in standard and experimental arms}

Prior to randomisation, based on pretreatment pelvic MRI, treating teams will be asked to declare whether they intend to administer postoperative adjuvant chemotherapy or not. This is to avoid an imbalance in the use of adjuvant chemotherapy between the two arms of the trial. Standard accepted indications will apply, for example, nodal involvement, T4 disease or extramural vascular invasion. Choice of chemotherapy will be in accordance with each site's current schedule and will be recorded on the case report form (CRF). In practice, little variation is anticipated.

\section{Primary objective}

To establish whether a future definitive trial comparing surgery (modern APER) alone with standard care (preoperative long course RT then surgery) is feasible.

\section{Secondary objectives}

- To set up and test the infrastructure necessary to perform a definitive trial.

- To test willingness of eligible participants to be randomised to an early surgery-alone arm (with adjuvant chemoradiation postsurgery if clinically indicated).

- To quantify the number of participants required for a definitive trial using appropriate outcome measures.

- To test the ability to recruit, consent and retain participants to the proposed intervention.

- To qualitatively explore reasons for non-recruitment.

- To test appropriateness and feasibility of collecting the proposed outcome measures for a full trial.

The primary outcome measure will establish whether a future definitive trial comparing surgery alone with current standard care is feasible.

All secondary outcomes will be rehearsed during the feasibility trial with a view to refining (1) the process and (2) the choice of outcomes for the main trial. The ability to collect the following clinical, pathological, QoL and health economic data will be tested.

A. EORTC QLQ-C30 and QLQ-CR29 QoL tools;

B. Health economic data collection tools (EuroQol5D-3L and Client Service Receipt Inventory);

C. Perioperative complication rates;

D. Rate of perineal wound healing at 3 months;

E. Time interval between surgery and initiation of adjuvant chemotherapy after surgery (days);
F. Data sets for radiological staging, pathological staging, RT delivery and surgical procedures to include:

- Distance of tumour to CRM (mm),

- Plane of mesorectal and sphincter/levator excision as proxy of surgical quality,

- Retrieval of macroscopic and cross-section photographs and histopathology slides for central review,

G. Detection of disease recurrence: local recurrence rate and systemic recurrence rate (distant metastases).

Patients who decline trial inclusion will be invited to participate in a qualitative semistructured interview to explore the reasons for this which will inform the definitive trial design.

\section{Site selection}

This feasibility trial will take place in colorectal units that have a high volume rectal cancer practice with highquality audited surgical results. Sites will be eligible to participate based on current case volumes, surgical quality (complete resection rates), clinical equipoise with regard to the prescription of neoadjuvant treatment for the participant entry criteria, RT target volume audits and ability to perform MRI and histopathology to the standard within the protocol.

\section{Participant selection}

Potential participants will be identified by their usual clinicians in the colorectal cancer multidisciplinary team meeting. They will be screened based on the MRI-defined inclusion criteria and suitability for RT (and chemotherapy if applicable). The inclusion and exclusion criteria are listed in box 1 .

If eligible, participants will receive study information from surgeon and oncologist and reinforced by a patient information sheet. Participants will be offered a minimum of 24 hours to consider enrolment before providing written informed consent. Informed consent will be obtained before trial-specific procedures are performed. A flow chart for the trial is included in figure 1.

\section{Randomisation}

Participants will be randomised 1:1 immediately after consent by a web-based method hosted by STU. From the results of this feasibility trial, we will establish any stratifying factors for randomisation in the future main trial.

\section{Blinding}

The trial statistician and independent radiologist and pathologist undertaking central review will be blinded to the treatment allocation until analysis has been undertaken and approval has been received by the Trial Steering Committee (TSC) to unlock the blinding.

\section{Data collection and management}

Data collection will be performed at baseline, after RT treatment (if standard care group), before, during and after surgery, and at the routine outpatient follow-up 


\section{Box 1 Participant selection}

\section{Inclusion criteria}

- Age 18 years or older;

- Histologically confirmed adenocarcinoma;

- Low rectal cancer defined as within $6 \mathrm{~cm}$ of anal verge on rigid sigmoidoscopy and considered to require abdominoperineal excision of the rectum rather than restorative procedure (anterior resection);

- Potentially resectable local disease by surgery alone with clear margins as determined by MRI;

- Radiologically measurable or clinically evaluable disease;

- Clinical disease stage (MRI \pm endorectal ultrasound):

- cT3a/b $(<5 \mathrm{~mm})$ disease within $6 \mathrm{~cm}$ of anal verge;

- For tumours at / below level of puborectalis through full thickness of muscularis propria (cT2) disease at level of puborectalis;

- Involvement of internal anal sphincter or intersphincteric space without extension into adjacent levator plate;

- TanyN1 (resectable) -irregular border or mixed signal on MRI;

- WHO performance status 0,1 , or 2 ;

- Blood values:

- Neutrophil count $\geq 1500 / \mathrm{mm}^{3}$;

- Platelets $\geq 100000 / \mathrm{mm}^{3}$;

- Haemoglobin $>80 \mathrm{~g} / \mathrm{L}$;

- Total bilirubin $\leq 1.5 \times$ upper limit of normal (ULN);

- Aspartate aminotransferase and alanine transaminase $\leq 3 \times \mathrm{ULN}$;

- Creatinine $\leq 1.5 \times U L N$;

- Negative pregnancy test;

- Patient of childbearing potential willing to employ adequate contraception;

- No other invasive malignancy $\leq 5$ years prior to registration;

- No concurrent disease that, in the judgement of the clinician obtaining informed consent, would make the patient inappropriate for entry into this trial;

- No chemotherapy within 5 years prior to registration;

- No prior pelvic radiation;

Able and willing to give informed consent to participate.

Exclusion criteria

- Preoperative chemoradiotherapy absolutely indicated, for example, predicted CRM involvement $(<1 \mathrm{~mm})$ by primary or nodal disease, or otherwise unresectable disease;

- cT3c or d $(>5 \mathrm{~mm})$;

- Adjacent organ involvement (prostate, seminal vesicles, sacrum or coccyx; T4b) requiring multivisceral resection/pelvic exenteration;

- For low tumours at level of puborectalis sling: lateral extension of tumour into external anal sphincter or beyond puborectalis sling into levator plate (pT4a);

- Extramural vascular invasion on MRI;

Early stage rectal cancer (T1, T2 above level of levators) unless node positive;

- Locally perforated disease (T4a);

- Fistulating disease (vagina, perianal skin, adjacent hollow organ);

- Disease extrusion through anus;

- cN2 disease;

- Lateral pelvic/para-aortic lymphadenopathy;

- Unresectable metastatic disease (M1; potentially resectable disease permitted);

- Previous pelvic radiotherapy;

- Unfit for major surgery;

- Pregnancy; Contraindication to MRI;

- Contraindication to standard chemotherapy (including drug interactions and glomerular filtration rate $<50 \mathrm{~mL} / \mathrm{min}$ at baseline);

- WHO performance status 3 or 4 or 5 ;

- Patients from vulnerable groups;

Unwilling to consent to trial participation.

visits at 1, 3, 6 and 12 months after their surgery. Participants will complete QoL questionnaires at the same intervals.

Pathology photographs, histological sections and MRI reports and scans will be collected.

Data will be collected on predesigned paper CRFs and entered into an electronic data capture system hosted by
STU by site staff. The trial database will have built in measures to assess data quality at time of input.

\section{Statistical analysis and sample size}

The sample size for this feasibility trial $(n=80)$ is the minimum number of participants considered necessary to test the processes of data collection, and based on the 
Figure 1 Trial flow chart.

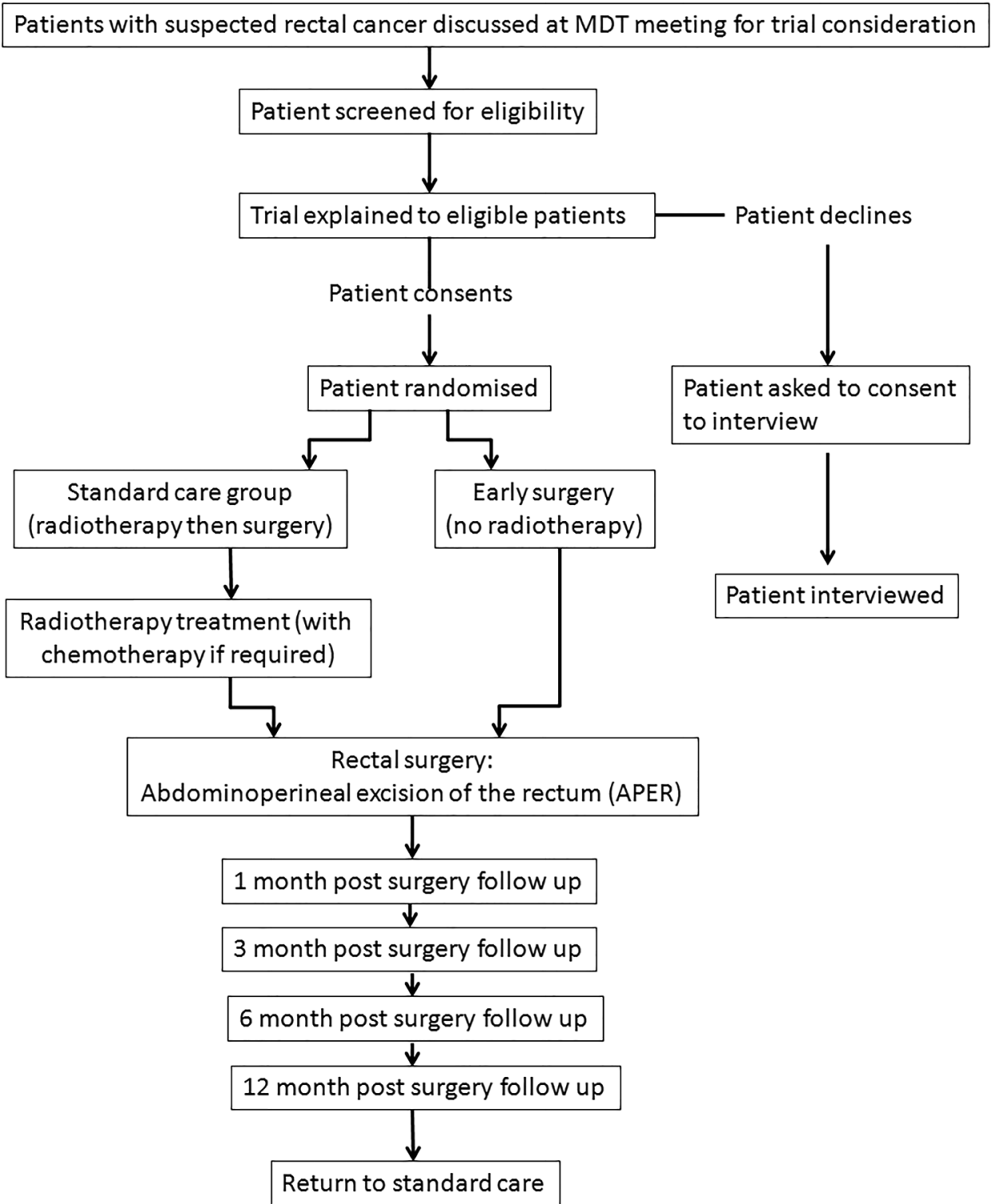

recommendations of Lancaster et $a l^{25}$ with respect to the number of patients required to yield meaningful estimates of parameters of interest.

For the purposes of this feasibility trial, we will use statistical test of hypothesis at 5\% level of significance with CIs using the key parameters of interest. These include:

- The proportion of participants who agree to be randomised.

- The proportion of participants undergoing surgery who experience complications/toxicities.

- The proportion of participants for whom we can collect outcomes for at 3, 6 and 12 months posttreatment (ie, follow-up rates):

- The response rates to the questionnaires.

- Adherence/compliance rates.

- The SD of the QoL measures that are proposed for the definitive trial.

- The effect size for the calculation of the sample size for the definitive trial.
The decision to proceed to a full definitive trial will be based on ACCEPT criteria. $^{26}$ Our specific criteria for progression to a full definitive trial are:

- Acceptable participant recruitment rates (>50\% eligible population).

- $>60 \%$ of randomised participants receive the treatment as allocated.

$>60 \%$ return rate of QoL questionnaires at 6 months.

- $>50 \%$ compliance with return of economic analysis data collection tools.

- Equivalent rates of CRM involvement are seen at 12 months.

\section{Safety measures}

Serious adverse events (SAEs) will be notified to STU within 24 hours and to the Research Ethics Committee (REC) within 15 days. A number of adverse events are expected for patients undergoing RT and colorectal surgery and will be exempted from reporting. 
Quality assurance

Radiotherapy

National Radiotherapy Trials Quality Assurance (RTTQA) Group will oversee the pretrial and on-trial QA, which can be streamlined on request. Benchmark cases (outlining and planning) will be assessed pretrial. Centres wishing to use IMRT (or volumetric modulated arc therapy) will be required to have completed the IMRT credentialing programme. On-treatment verification by imaging the first three fractions of treatment and weekly thereafter is mandatory. The first study patient will have prospective review of contouring and planning in real time as a minimum.

\section{MRI}

Sagittal T2-weighted turbo spin echo, large field of view (FOV) axial T2 images of whole pelvis L5/S1, highresolution axial T2-weighted images and high-resolution coronal T2-weighted images will be obtained at baseline. The protocol mandates $3 \mathrm{~mm}$ slices with a $16-18 \mathrm{~cm}$ FOV to optimise image quality. Images will be transferred securely via image exchange portal or equivalent system for central review.

\section{Surgery}

Sites will be required to demonstrate satisfactory results of APER surgery pretrial. The surgical technique is protocolised and requires wide excision of the pelvic floor. Surgeons will declare their intended planes of excision presurgery on a template which will be compared with actual pathological plane of excision.

\section{Pathology}

A standardised protocol based on RCPath guidelines will be used. Specimen photography is mandatory (whole intact specimen and cross-sectional slices). Quality of the mesorectal and sphincter/levator excision will be assessed locally and then independently and blindly assessed at the University of Leeds according to the protocol.

\section{Ethics and dissemination}

The trial will be performed in accordance with the principles of the Declaration of Helsinki and Good Clinical Practice. The trial is approved by the Regional National Health Service (NHS) Ethics Committee and local hospital Research and Development permissions. Written informed consent will be obtained from all participants. All SAEs will be reported to STU, the ethics committee and all sites will be notified of events.

It is recognised that there is a substantial body of evidence in favour of preoperative long course RT in locally advanced rectal cancer to minimise local recurrence. Risk factor for this are involved surgical resection margins, T4 disease, N2 disease and extramural venous invasion. Certain tumour characteristics mandate the use of neoadjuvant treatment, particularly predicted surgical resection margin involvement, so these patients will not be eligible for this trial. The inclusion criteria have been carefully selected to focus on the borderline group of patients where high-quality early surgery alone may well be sufficient treatment, in conjunction with appropriate adjuvant therapies.

To minimise patient risk, potential sites will be required to demonstrate evidence of high standards of imaging, RT, pathology and surgical quality before eligibility. On-trial QA will uphold these standards.

The trial will be scrutinised throughout by an independent TSC who will also assume the role of the Data Monitoring Committee. Particular attention will be given to margin involvement rates. In the event of high-risk pathological features for local pelvic recurrence being detected after surgery $(\mathrm{CRM} \leq 1 \mathrm{~mm})$, patients will be offered adjuvant postoperative chemoradiation.

Publication of trial results in peer-reviewed journals will include named members of the Trial Management Group (TMG) meeting the three criteria of scholarship (design, execution, analysis and/or interpretation of the data), authorship (drafting, reviewing and revision of the manuscript) and approval (approving the manuscript to be published). Results will be disseminated through the funding body and through Cancer Research UK. Participants in the trial will be given a copy of the results. A final report will be written by the TMG for the funding body and the REC.

\section{DISCUSSION}

The need to improve pretreatment risk stratification was further highlighted by Gunderson $e t a l^{27}$ who suggested patients at intermediate risk of LR (T1-2N1, T3N0) might be overtreated with neoadjuvant (C)RT. It is recognised that high-resolution MRI staging can accurately predict those patients with good prognosis stage III rectal tumours suitable for treatment with highquality surgery alone. ${ }^{22}$ The Mayo Clinic group reported just $5.5 \%$ local recurrence rate in which APER comprised $38 \%$ of the series in a cohort of 655 patients treated without RT, in which over $20 \%$ had stage III rectal tumours. ${ }^{28}$ Data from Swansea reports equivalent rates of margin involvement, local recurrence and survival in T3/T4 node positive rectal cancers in which neoadjuvant treatment was reserved for predicted margin involvement and significant nodal disease, ${ }^{29}$ which is confirmed by longitudinal analysis of this selective approach to RT. $^{30}$ Given the improvements in CRM rates and local recurrence with the modern APE, it is timely to scrutinise the role of neoadjuvant RT in this setting. Rigorous prospective studies are required not only to determine which subsets of stage II/III rectal cancer benefit from neoadjuvant therapy, but also to define the contribution of modern surgery to local disease control. Authorities support the need for trials in stage II and III rectal cancer to minimise overtreatment with RT. $^{31}{ }^{32}$ The potential patient benefits of having high-quality surgery without RT include 
improved QoL, reduced complications and long-term morbidity and earlier access to postoperative adjuvant systemic chemotherapy. Economic advantage from chemoradiotherapy avoidance is estimated to be $£ 6690$ per patient without including the cost of managing related long-term complications (local data). This research is integral to the future management of patients with rectal cancer worldwide and carries international clinical importance with the prospect of changing current guidelines.

\section{Author affiliations}

${ }^{1}$ Department of Colorectal Surgery, Singleton Hospital, Swansea, UK

${ }^{2}$ Swansea Trials Unit, Swansea University, Swansea, UK

${ }^{3}$ South West Wales Cancer Centre, Singleton Hospital, Swansea, UK

${ }^{4}$ Department of Colorectal Surgery, St Marks Hospital, London, UK

${ }^{5}$ Department of Colorectal Surgery, Royal Victoria Infirmary, Newcastle, UK

${ }^{6}$ Department of Colorectal Surgery, Western General Hospital, Edinburgh, UK

${ }^{7}$ Department of Colorectal Surgery, Royal Cornwall Hospital, Truro, UK

${ }^{8}$ Department of Colorectal Surgery, St Vincent's Hospital, Dublin, Ireland

${ }^{9}$ Department of Oncology, North Wales Cancer Treatment Centre, Rhyl, UK

${ }^{10}$ Pathology and Tumour Biology, Leeds Institute of Oncology and Pathology, Wellcome Trust Brenner Building, St James Hospital, Leeds, UK

${ }^{11}$ Department of Radiology, Royal Marsden Hospital, London, UK

${ }^{12}$ Swansea Centre for Health Economics, Swansea University, Swansea, UK

${ }^{13}$ Involving People Network, Health and Care Research Wales

Twitter Follow Kymberley Thorne at @kymberleythorne

Contributors DAH and JB are responsible for the idea for the trial. DAH, KT, $\mathrm{HH}, \mathrm{SI}, \mathrm{GH}, \mathrm{OH}, \mathrm{SG}, \mathrm{IJ}, \mathrm{PC}, \mathrm{MD}, \mathrm{MF}, \mathrm{DCW}, \mathrm{SG}, \mathrm{PQ}, \mathrm{NW}, \mathrm{GB}, \mathrm{DF}, \mathrm{AB}$ and JB have made substantial contributions to the conception and design of the work and subsequent protocol revisions; drafted the manuscript and/or provided critical revision; approved the version submitted for publication; agree to be accountable for all aspects of the work in ensuring that questions related to the accuracy or integrity of any part of the work are appropriately investigated and resolved.

Funding This work is supported by Bowel Disease Research Foundation and endorsed by Cancer Research UK (CRUKE/14/050). PQ and NW are funded by Yorkshire Cancer Research. DAH is supported by a Health and Care Research Wales Clinical Research Time award. Sponsor: Abertawe Bro Morgannwg University Health Board will assume overall responsibility for the trial as sponsor.

Competing interests None declared.

Ethics approval Wales REC6.

Provenance and peer review Not commissioned; externally peer reviewed.

Open Access This is an Open Access article distributed in accordance with the Creative Commons Attribution Non Commercial (CC BY-NC 4.0) license, which permits others to distribute, remix, adapt, build upon this work noncommercially, and license their derivative works on different terms, provided the original work is properly cited and the use is non-commercial. See: http:// creativecommons.org/licenses/by-nc/4.0/

\section{REFERENCES}

1. Cancer Research UK. Bowel cancer incidence statistics. Secondary Bowel cancer incidence statistics. 2016. http://www.cancerresearchuk. org/health-professional/cancer-statistics/statistics-by-cancer-type/ bowel-cancer/incidence

2. De Caluwe L, Van Nieuwenhove Y, Ceelen WP. Preoperative chemoradiation versus radiation alone for stage II and III resectable rectal cancer. Cochrane Database Syst Rev 2013;2: CD006041.

3. National Institute for Health and Clinical Excellence. Colorectal cancer: the diagnosis and management of colorectal cancer (CG131). National Institute for Health and Clinical Excellence, 2011.
4. National Comprehensive Cancer Network. NCCN guidelines for treatment of cancer by site. Secondary NCCN guidelines for treatment of cancer by site. 2016. http://www.nccn.org/professionals/ physician_gls/f_guidelines.asp

5. Glimelius B, Tiret E, Cervantes A, et al. Rectal cancer: ESMO Clinical Practice Guidelines for diagnosis, treatment and follow-up. Ann Oncol 2013:24(Suppl 6):vi81-8.

6. Augestad KM, Lindsetmo RO, Stulberg J, et al. International preoperative rectal cancer management: staging, neoadjuvant treatment, and impact of multidisciplinary teams. World J Surg 2010;34:2689-700.

7. Bruheim K, Guren MG, Skovlund E, et al. Late side effects and quality of life after radiotherapy for rectal cancer. Int $J$ Radiat Oncol Biol Phys 2010;76:1005-11.

8. Rothenberger DA, Akbari R, Baxter NN. Are we overtreating some patients with rectal cancer? Oncology 2004;18:1789-96; discussion 96, 99-804.

9. Swellengrebel HA, Marijnen CA, Verwaal VJ, et al. Toxicity and complications of preoperative chemoradiotherapy for locally advanced rectal cancer. Br J Surg 2011;98:418-26.

10. Pachler J, Wille-Jørgensen P. Quality of life after rectal resection for cancer, with or without permanent colostomy. Cochrane Database Syst Rev 2012;12:CD004323.

11. Glynne-Jones R. Neoadjuvant treatment in rectal cancer: do we always need radiotherapy-or can we risk assess locally advanced rectal cancer better? Recent Results Cancer Res 2012;196:21-36.

12. Quirke $P$, Dixon MF. The prediction of local recurrence in rectal adenocarcinoma by histopathological examination. Int $J$ Colorectal Dis 1988;3:127-31.

13. Health and Social Care Information Centre. National Bowel Cancer Audit report 2011. Secondary National Bowel Cancer Audit report 2011. 2011. http://www.hscic.gov.uk/catalogue/PUB02576/ nati-clin-audi-supp-prog-bowe-canc-2011-rep1.pdf

14. Health and Social Care Information Centre. National Bowel Cancer Audit report 2008. Secondary National Bowel Cancer Audit report 2008. 2008. http://www.hscic.gov.uk/catalogue/PUB02603/ nati-clin-audi-supp-prog-bowe-canc-2008-rep.pdf

15. Holm $T$, Ljung $A$, Häggmark $T$, et al. Extended abdominoperineal resection with gluteus maximus flap reconstruction of the pelvic floor for rectal cancer. Br J Surg 2007;94:232-8.

16. Nagtegaal ID, van de Velde CJ, Marijnen CA, et al. Low rectal cancer: a call for a change of approach in abdominoperineal resection. J Clin Oncol 2005;23:9257-64.

17. Moran BJ, Holm T, Brannagan G, et al. The English national low rectal cancer development programme: key messages and future perspectives. Colorectal Dis 2014;16:173-8.

18. West NP, Finan PJ, Anderin C, et al. Evidence of the oncologic superiority of cylindrical abdominoperineal excision for low rectal cancer. J Clin Oncol 2008;26:3517-22.

19. Sauer R, Becker H, Hohenberger W, et al. Preoperative versus postoperative chemoradiotherapy for rectal cancer. $N$ Engl J Med 2004;351:1731-40.

20. Bosset JF, Calais G, Mineur L, et al. Enhanced tumorocidal effect of chemotherapy with preoperative radiotherapy for rectal cancer: preliminary results-EORTC 22921. J Clin Oncol 2005;23: 5620-7.

21. Quirke P, Steele R, Monson J, et al. Effect of the plane of surgery achieved on local recurrence in patients with operable rectal cancer: a prospective study using data from the MRC CR07 and NCIC-CTG CO16 randomised clinical trial. Lancet 2009;373:821-8.

22. Taylor FG, Quirke P, Heald RJ, et al. Preoperative high-resolution magnetic resonance imaging can identify good prognosis stage I, II, and III rectal cancer best managed by surgery alone: a prospective, multicenter, European study. Ann Surg 2011;253: 711-19.

23. Battersby NJ, How P, Moran B, et al. Prospective validation of a low rectal cancer magnetic resonance imaging staging system and development of a local recurrence risk stratification model: the MERCURY II study. Ann Surg 2015;263:751-60.

24. Cancer National Specialist Advisory Group. Wales Bowel Cancer Audit report for patients diagnosed April 2009-March 2010 and August 2010-July 2011. Secondary Wales Bowel Cancer Audit report for patients diagnosed April 2009-March 2010 and August 2010-July 2011. 2013. http://www.wales.nhs.uk/sites3/Documents/ 322/Welsh\%20Bowel\%20Cancer\%20Audit 2009_11_.pdf

25. Lancaster GA, Dodd S, Williamson PR. Design and analysis of pilot studies: recommendations for good practice. J Eval Clin Pract 2004:10:307-12.

26. Charlesworth G, Burnell K, Hoe J, et al. Acceptance checklist for clinical effectiveness pilot trials: a systematic approach. BMC Med Res Methodol 2013;13:78. 
27. Gunderson LL, Callister M, Marschke R, et al. Stratification of rectal cancer stage for selection of postoperative chemoradiotherapy: current status. Gastrointest Cancer Res 2008;2:25-33.

28. Mathis KL, Larson DW, Dozois EJ, et al. Outcomes following surgery without radiotherapy for rectal cancer. Br J Surg 2012;99:137-43.

29. Williamson JS, Jones HG, Davies M, et al. Outcomes in locally advanced rectal cancer with highly selective preoperative chemoradiotherapy. Br J Surg 2014;101:1290-8.
30. Davies M, Harries D, Hirst G, et al. Local recurrence after abdomino-perineal resection. Colorectal Dis 2009;11:39-43.

31. Glynne-Jones R, Harrison M, Hughes R. Challenges in the neoadjuvant treatment of rectal cancer: balancing the risk of recurrence and quality of life. Cancer Radiother 2013;17: 675-85.

32. Wibe A, Law WL, Fazio V, et al. Tailored rectal cancer treatment-a time for implementing contemporary prognostic factors? Colorectal Dis 2013;15:1333-42. 